\title{
Role of Adjuvant Treatment in Sinonasal Mucosal Melanoma
}

\author{
Moran Amit ${ }^{1, *}$ Samantha Tam ${ }^{1, *}$ Ahmed S. Abdelmeguid ${ }^{1,2}$ Michael E. Kupferman ${ }^{1}$ Shirley Y. Su ${ }^{1}$ \\ Shaan M. Raza ${ }^{3}$ Franco DeMonte $^{3}$ Ehab Y. Hanna ${ }^{1}$
}

${ }^{1}$ Department of Head and Neck Surgery, Division of Surgery, The University of Texas MD Anderson Cancer Center, Houston, Texas, United States

${ }^{2}$ Department of Otolaryngology Head and Neck Surgery, Faculty of Medicine, Mansoura University, Egypt

${ }^{3}$ Department of Neurosurgery, Division of Surgery, The University of

Texas MD Anderson Cancer Center, Houston, Texas, United States

J Neurol Surg B 2017;78:512-518.
Address for correspondence Ehab Y. Hanna, MD, Department of Head and Neck Surgery, Division of Surgery, The University of Texas MD Anderson Cancer Center, 1515 Holcombe Blvd, Unit 1445, Houston, TX 77030, United States (e-mail: EYHanna@mdanderson.org).

\begin{abstract}
Keywords

- mucosal

- melanoma

- sinonasal

- skull base

- recurrence

- chemotherapy

- radiation

- survival

Purpose Sinonasal mucosal melanoma (SNMM) is a locally aggressive tumor. This study aimed to define the role of adjuvant treatment and its association with survival outcomes of SNMM.

Methods This retrospective study investigated 152 patients with SNMM treated between 1991 and 2016 in MD Anderson Cancer Center. Patients were divided into the following treatment groups: surgery alone, surgery with postoperative radiotherapy (PORT), surgery with postoperative chemoradiation (POCRT), and induction chemotherapy followed by surgery and PORT. Overall survival (OS), disease-specific survival, and relapse-free survival were compared. Survival between the groups was compared using univariate and multivariate analyses.

Results The median follow-up was 28 months (range: 2-220 months). Five-year OS rates were $39,42,47$, and $27 \%$ for the surgery only, PORT, POCRT, and neoadjuvant chemotherapy groups, respectively ( $\log$ rank $p=0.73$ ). Distant metastasis was the most common form of treatment failure and occurred in 59 (39\%) patients. Five-year distant metastasis rates were 51,45 , and $58 \%$ for patients treated with surgery alone, PORT, and POCRT, respectively (log rank $p=0.21$ ) but unable to be estimated in the neoadjuvant chemotherapy group due to low OS rates. Multivariate analysis demonstrated tumor site (hazard ratio $[\mathrm{HR}]=2.32,95 \%$ confidence interval $[\mathrm{Cl}]=1.24$ 4.15) and smoking status ( $\mathrm{HR}=1.77,95 \% \mathrm{Cl}=1.02-3.1)$ to be significant prognostic factors for survival.

Conclusion Tumor site and smoking status were significant prognosticators in SNMM. A high rate of distant metastatic disease suggests that further investigation into novel, systemic therapies is required to improve outcomes in this disease entity.
\end{abstract}

\footnotetext{
These authors contributed equally to the study.
}

received

March 13, 2017

accepted after revision

June 10, 2017

published online

July 31,2017 (c) 2017 Georg Thieme Verlag KG

Stuttgart · New York
DOI https://doi.org/ 10.1055/s-0037-1604350. ISSN 2193-6331. 


\section{Introduction}

Melanomas are tumors derived from melanocytes and most commonly occur in the skin. ${ }^{1}$ However, melanomas can also occur on mucosal line surfaces due to melanocyte migration as neuroectodermal derivatives into the ectodermal tissue. ${ }^{2}$ Mucosal melanoma represents $1.3 \%$ of all melanomas, with 50 to $55 \%$ occurring in the head and neck. ${ }^{3}$ The sinonasal cavity is the most common site for head and neck mucosal melanoma but is still a rare tumor, accounting for $1 \%$ of all melanomas in the head and neck. ${ }^{3,4}$

As with cutaneous melanomas, surgical excision is the primary treatment of choice. ${ }^{4}$ However, especially with sinonasal mucosal melanomas (SNMMs), anatomic considerations may make obtaining adequate margins difficult, if not impossible. As such, adjuvant treatment is an important consideration in the treatment of this entity. Studies have previously attempted to quantify the utility of adjuvant therapy in sinonasal melanomas. A meta-analysis conducted by Li et al demonstrated no survival benefits for postoperative radiotherapy in head and neck mucosal melanomas or SNMMs. ${ }^{5}$ However, a subgroup analysis showed improved locoregional control in all mucosal head and neck melanomas, but the analysis was not completed only for SNMMs. The effect of systemic therapy is less defined due to the variety of regimens available. ${ }^{6-8}$ This study aims to describe the effects of postoperative radiotherapy and chemotherapy in patients treated primarily with surgery and identify risk factors to aid in decision making about the extent of adjuvant therapy.

\section{Methods}

This retrospective review was conducted at the University of Texas MD Anderson Cancer Center after obtaining institutional review board approval (Protocol RCR04-0636). One hundred and seventy consecutive patients who presented between August 1991 and July 2016 to our institution were retrospectively reviewed.

Patients were included if (1) the diagnosis of mucosal melanoma was pathologically confirmed at our institution, (2) the primary site of the tumor was in the sinonasal cavity, and (3) the primary modality of treatment was surgery. Exclusion criteria were patients in whom no treatment data could be collected, patients who underwent primary radiotherapy or chemotherapy, or patients receiving therapy for palliative care only. Patient demographics (age, gender, diagnostic date, smoking, and alcohol intake), staging, tumor characteristics, treatment modalities, pathologic data (tumor thickness, ulceration, perineural and lymphovascular invasion, bony invasion, and number of mitotic figures), and clinical outcomes were collected. The histopathological specimens of the tumors were evaluated by a certified head and neck pathologist in each center according to the current guidelines for the histopathological assessment of head and neck cancer carcinomas. $^{9}$ All staging was completed according to the

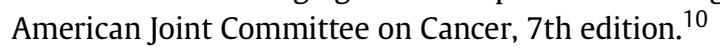

All patients were treated primarily with surgery. To compare outcomes, patients were divided into four groups depending on adjuvant therapy: Group 1, surgery with no adjuvant therapy; group 2, postoperative radiotherapy (PORT); group 3, postoperative chemoradiotherapy (POCRT); and group 4, induction chemotherapy and postoperative PORT. Patients undergoing PORT were additionally divided into two groups: (1) those undergoing conventionally fractionated radiotherapy, defined as 1.8 to $2.0 \mathrm{~Gy} /$ fraction delivered 5 days per week and (2) those undergoing hypofractionation, defined as $5.0 \mathrm{~Gy} /$ fraction delivered less than 5 days per week. Primary outcome measures were overall survival(OS), disease-specific survival(DSS), and relapse-free survival (RFS). Index date for all survival outcomes was date of initiation of treatment. Relapse was defined as any evidence of disease following the date of completion of initial treatment. Distant metastasis failure rate was defined as time from initiation of treatment to presence distant metastasis.

Descriptive statistics were obtained for all baseline variables. Student's $t$-test was used to compare all continuous variables and the chi-squared test was used for categorical variables. The Kaplan-Meier method was employed for all survival analyses. The log-rank test was used to compare survival outcomes between adjuvant treatment groups. Univariate and multivariate analyses with the Cox Proportional Hazards Model were used to identify possible indicators for adjuvant therapy. All statistical tests were two tailed. Alpha was set to 0.05 for significance. All statistical tests were completed on SAS JMP Pro version 12.1.0 (SAS Institute, Cary, North Carolina, United States).

\section{Results}

A total of 152 patients met inclusion and exclusion criteria and were included for analysis. The patients ranged in age from 34 to 91 years (median: 64 years). Follow-up ranged from 2 to 220 months (median: 28 months). Clinical and pathological characteristics according to treatment groups are described in -Table 1. One hundred nineteen patients $(78 \%)$ had nasal cavity mucosal melanoma, 32 patients $(21 \%)$ had paranasal sinus mucosal melanoma, and 1 patient had a tumor whose epicenter could not be determined. Ninety-seven patients (64\%) had T3 disease and 55 patients (36\%) had T4a/b disease. Nodal metastases were present in 12 patients (8\%).

Fifty-seven patients (38\%) underwent surgical resection alone. PORT was administered in 81 patients (53\%), and of these patients, $8(10 \%)$ underwent POCRT. In patients receiving radiation, hypofractionated radiotherapy was administered in 24 patients (30\%) and conventionally fractionated radiotherapy was administered in 57 patients (70\%). Neoadjuvant chemotherapy with cisplatin, vinblastine, and dacarbazine (CVD) and PORT was administered in 14 patients (9\%).

Five-year OS and DSS were 41 and 61\%, respectively (-Fig. 1). Five-year OS rates were $39 \%$ for patients treated with surgery alone, $42 \%$ for patients undergoing PORT, $47 \%$ for patient treated with POCRT, and $27 \%$ for patients treated with neoadjuvant chemotherapy followed by surgery and PORT ( - Fig. 2a, log rank $p=0.73$ ). Their DSS rates were 66, 61,64 , and $43 \%$, respectively (-Fig. $\mathbf{2 b}, \log$ rank $p=0.52$ ). 
Table 1 Baseline patient demographics and clinical characteristics

\begin{tabular}{|c|c|c|c|c|c|c|}
\hline Variable & & $\begin{array}{l}\text { Surgery } \\
(n=57)\end{array}$ & $\begin{array}{l}\text { Neoadjuvant chemotherapy } \\
\text { and POCRT }(n=14)\end{array}$ & $\begin{array}{l}\text { Surgery and } \\
\text { PORT }(n=73)\end{array}$ & $\begin{array}{l}\text { Surgery and } \\
\text { POCRT }(n=8)\end{array}$ & $p$-Value \\
\hline Age (y) & Mean \pm SD & $63 \pm 13$ & $59 \pm 12$ & $65 \pm 11$ & $61 \pm 14$ & 0.28 \\
\hline \multirow[t]{2}{*}{ Gender } & Male & $25(44)$ & $5(36)$ & $33(45)$ & $2(25)$ & 0.66 \\
\hline & Female & $32(56)$ & $9(64)$ & $40(55)$ & $6(75)$ & \\
\hline \multirow[t]{2}{*}{ Site } & Nasal cavity & $47(82)$ & $5(36)$ & $61(84)$ & $6(75)$ & 0.003 \\
\hline & Paranasal sinuses & $10(18)$ & $9(64)$ & $12(16)$ & $2(25)$ & \\
\hline \multirow[t]{3}{*}{ T classification } & 3 & $36(63)$ & $5(36)$ & $51(70)$ & $4(50)$ & \multirow[t]{3}{*}{0.07} \\
\hline & $4 a$ & $20(35)$ & $8(57)$ & $23(30)$ & $3(38)$ & \\
\hline & $4 \mathrm{~b}$ & $1(2)$ & $1(7)$ & 0 & $1(12)$ & \\
\hline \multirow[t]{2}{*}{ N classification } & No & $48(84)$ & $11(78)$ & $69(94)$ & $6(75)$ & \multirow[t]{2}{*}{0.09} \\
\hline & $\mathrm{N}+$ & $9(16)$ & $3(22)$ & $4(6)$ & $2(25)$ & \\
\hline \multirow[t]{2}{*}{ Mitosis } & $<1 \mathrm{~mm}^{2}$ & $41(72)$ & $9(64)$ & $55(75)$ & $5(63)$ & \multirow[t]{2}{*}{0.76} \\
\hline & $\geq 1 \mathrm{~mm}^{2}$ & $16(28)$ & $5(36)$ & $18(25)$ & $3(37)$ & \\
\hline \multirow[t]{2}{*}{ Ulceration } & Present & $43(75)$ & $11(79)$ & $59(80)$ & $5(63)$ & 0.67 \\
\hline & Absent & $14(25)$ & $3(21)$ & $14(20)$ & $3(37)$ & \\
\hline Follow-up (mo) & Mean \pm SD & $44 \pm 5$ & $37 \pm 14$ & $57 \pm 6$ & $55 \pm 19$ & 0.39 \\
\hline
\end{tabular}

Abbreviations: CRT, chemoradiation therapy; POCRT, postoperative chemoradiation; PORT, postoperative radiotherapy; RT, radiotherapy; SD, standard deviation.

During follow-up, 101 (66\%) patients experienced recurrence and the five-year RFS rate was $42 \%$. Of these, 59 (58\%) had distant metastasis. Five-year RFS was $25,22,34$, and $44 \%$ for patients treated with surgery alone, PORT, POCRT, and neoadjuvant chemotherapy followed by surgery and radiation, respectively ( $\mathbf{- F i g .} \mathbf{2 c}, \log$ rank $p=0.41$ ). Five-year distant metastasis rates were 51,45 , and $58 \%$ for patients treated with surgery alone, PORT, and POCRT, respectively ( - Fig. 2d, $\log$ rank $p=0.21$ ). Distant metastasis failure rate for patients treated with neoadjuvant chemotherapy followed by surgery and radiation could not be estimated due to low OS rates.

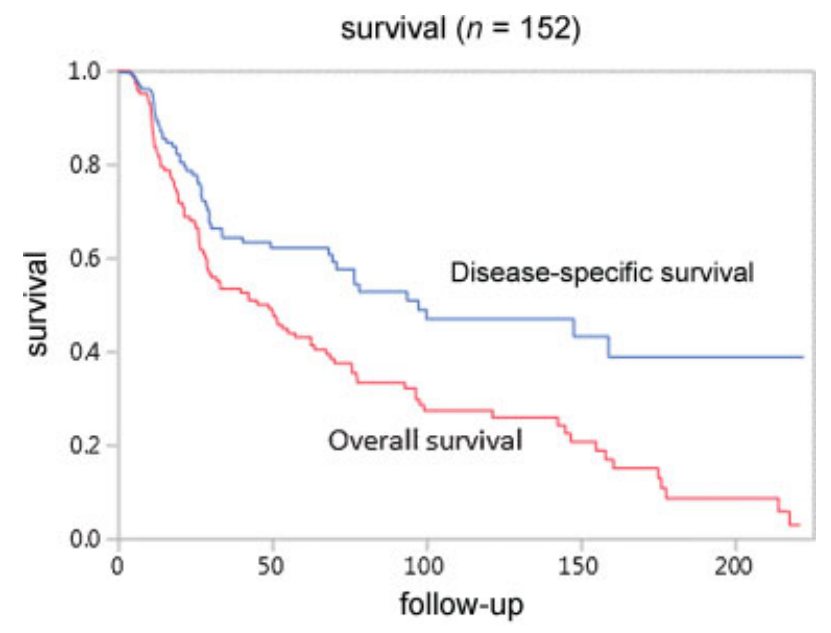

Fig. 1 Kaplan-Meier analysis of outcome in patients with sinonasal mucosal melanoma.
The impact of fractionation schedule on outcome was assessed. Patients treated with postoperative hypofractionated radiotherapy had a five-year OS of 35\% compared with $42 \%$ for patients treated with conventional fractionation ( - Fig. 3a, $\log$ rank $p=0.47$ ). Five-year DSS of patients treated with postoperative hypofractionated radiotherapy was $51 \%$ compared with $58 \%$ for patients treated with conventional fractionation ( - Fig. $\mathbf{3 b}, \log$ rank $p=0.58$ ). Patients treated with hypofractionated PORT had a five-year RFS of $5 \%$ compared with $28 \%$ in those treated with conventional fractionation. However, this difference did not reach statistical significance ( $\boldsymbol{- F i g . ~ 3 c , ~} \log$ rank $p=0.19$ ).

Univariate analysis revealed that age $\geq 65$ years, surgical margin status, and tumor site were significant predictors of five-year OS and DSS (- Table 2). Smoking status was significant for OS (hazard ratio [HR] $=1.77,95 \%$ confidence interval [CI]: 1.0-3.1) but not DSS (HR $=1.46,95 \% \mathrm{CI}$ : $0.74-2.3)$. In the multivariate model, the single independent predictor of both OS and DSS was tumor site (HR $=2.32,95 \% \mathrm{CI}: 1.24-4.15$ and $\mathrm{HR}=2.4,95 \% \mathrm{CI}: 1.13-4.99$, respectively). Smoking status was an independent prognostic factor for OS ( $\mathrm{HR}=1.77,95 \% \mathrm{CI}$ : 1.02-3.1; - Table 2). The type of adjuvant treatment had no significant influence on outcome.

\section{Discussion}

Cutaneous melanoma has traditionally been regarded as relatively insensitive to radiation and chemotherapy. However, there are no randomized trials studying the role of these treatment modalities in mucosal melanomas. In this study, we present the outcomes of patients treated in a single institute for SNMM. Our data show no significant survival 

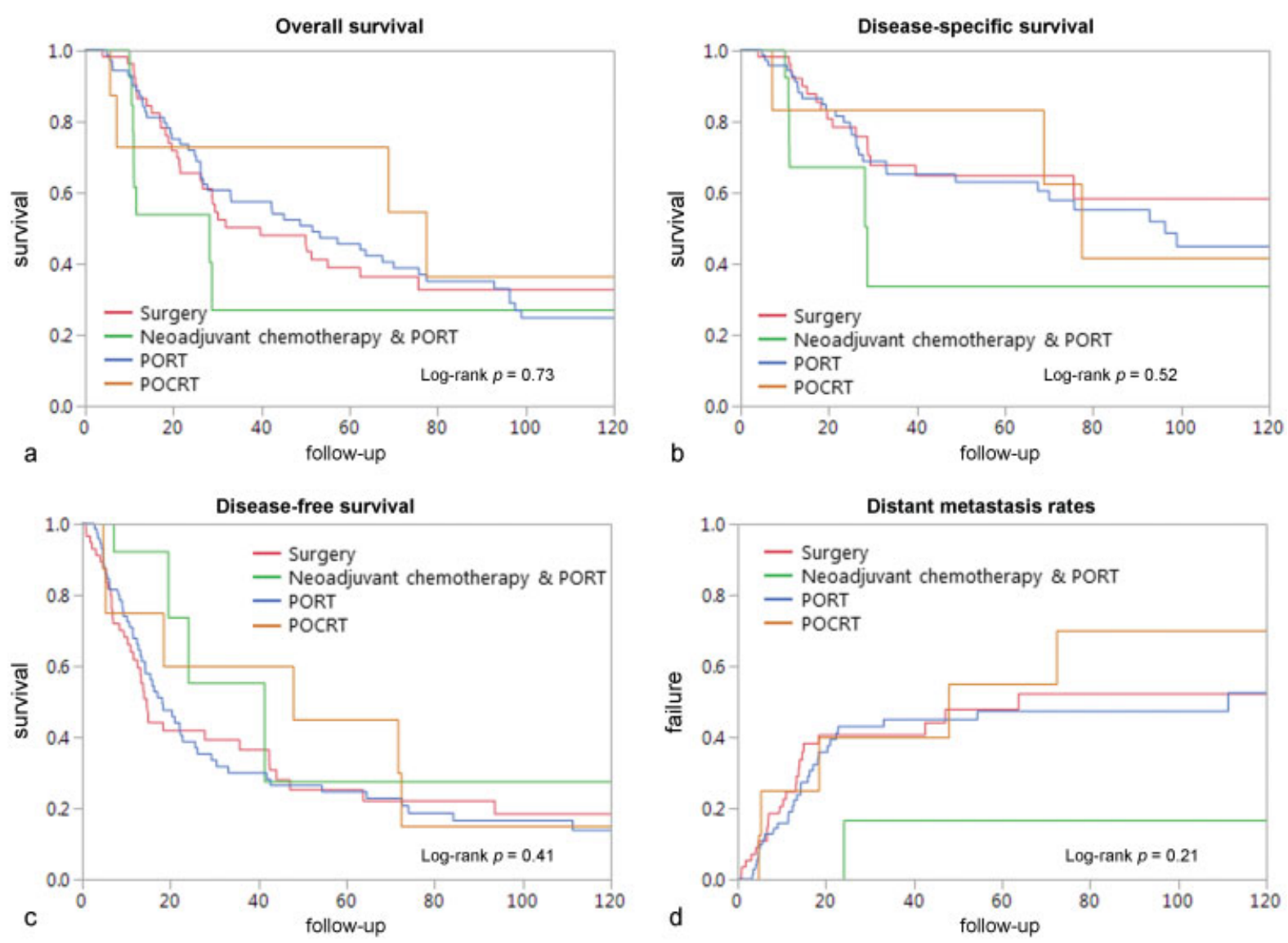

Fig. 2 Ten-year Kaplan-Meier analysis of outcome of patients with sinonasal mucosal melanoma according to treatment modality by (a) overall survival, (b) disease-specific survival, (c) disease-free survival, and (d) rate of distant metastasis.

benefits for any of the adjuvant treatment regimen. However, we found a significantly worse survival in patients with SNMM originating in the paranasal sinuses compared with the nasal cavity.

Surgical resection with clear margins remains the treatment of choice in mucosal melanomas. Radiation therapy has typically been indicated as an adjuvant treatment based on several retrospective studies that demonstrated an improved local control in patients treated with surgery and radiation therapy compared with those treated with surgery alone. ${ }^{11-14}$ However, none of these studies showed a significant improvement in OS in patients treated with PORT. Similarly, multivariate analysis of our data failed to demonstrate any significant improvement in either OS or DSS in patients treated with PORT compared with those who had only surgery. Some authors explain the lack of survival benefits by a higher rate of distant metastasis related to the more advanced stage of disease in patients treated with adjuvant radiation. ${ }^{15}$ Still, our data show no difference neither in survival nor in distant metastasis rates in patients treated locally with PORT or systemically with adjuvant chemotherapy.

A major limitation of previous studies is their heterogeneity. All published studies to date that showed a significant benefit for radiotherapy in head and neck mucosal melanoma included patients with SNMM and mucosal melanoma of other sites in the upper aerodigestive tract. The National
Comprehensive Cancer Network (NCCN) guidelines identify SNMM as a separate entity with a distinct therapeutic strategy with regard to mucosal melanomas of the head and neck. Furthermore, multivariate analysis of our data revealed that the tumor site within the sinonasal region is an independent prognostic factor. Taken together, optimizing local control by PORT can be advocated to mainly preserve patient's quality of life. However, these gains can be achieved in some patients by repeated surgeries with good functional results. Therefore, radiation-related toxicities should be weighed against the potential minimal benefits in patients with limited disease. ${ }^{16}$

Treatment may also vary among different institutions. As a high-volume center, all SNMM patients treated in MD Anderson Cancer Center are evaluated by a multidisciplinary team that includes head and neck surgeons and radiation and medical oncologists. When needed, neurosurgeons, ophthalmologists, and reconstructive surgeons are consulted. For localized disease, surgical resection with negative margins is the main treatment modality. Adjuvant radiation is administered in patients with locally advanced disease (i.e., skull base, bone, dura, brain, eye, or palate invasion), paranasal sinus involvement, and adverse pathological features (e.g., perineural invasion, nodal spread with extra nodal extension). Indications for chemotherapy are less well defined in nonmetastatic (M0) patients, and the role of induction 

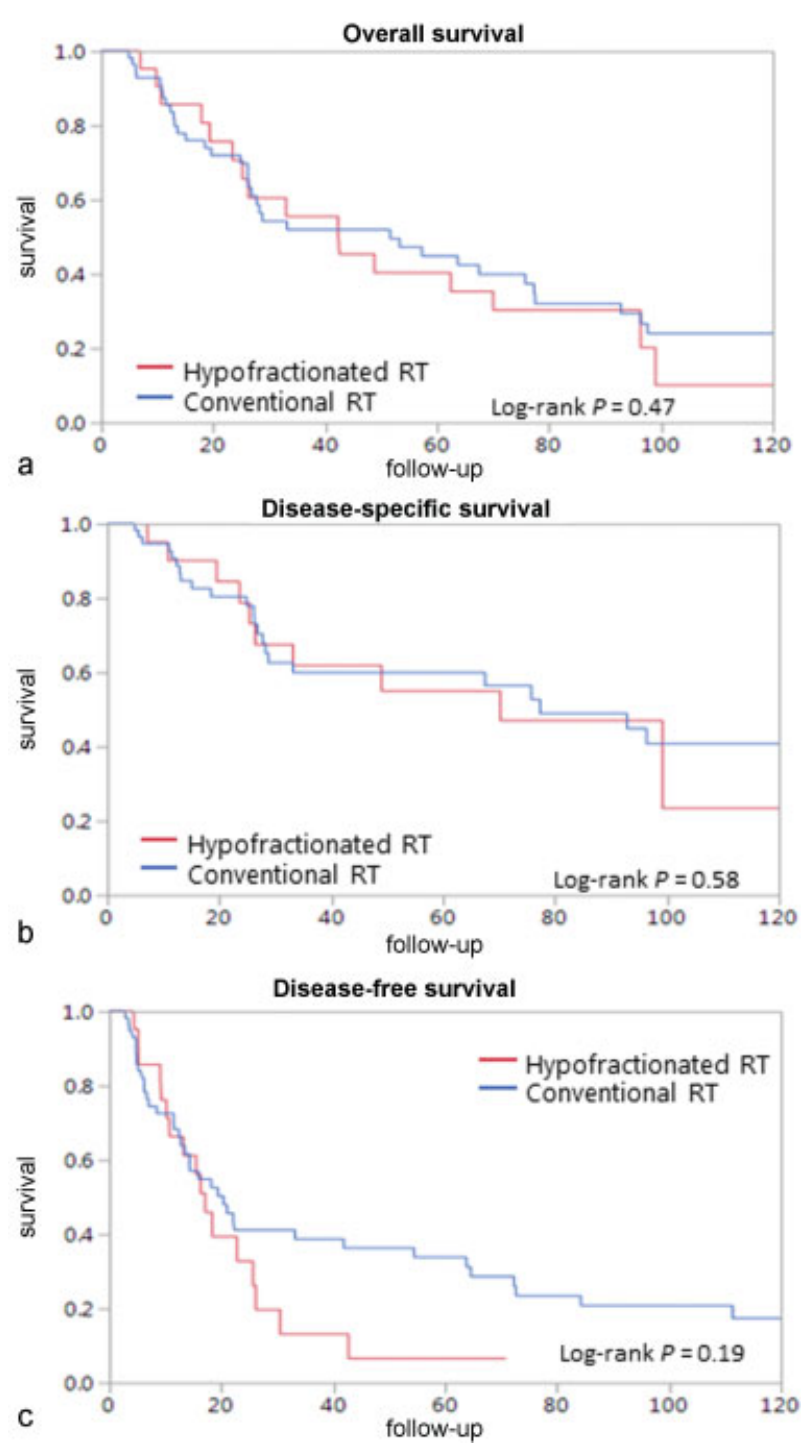

Fig. 3 Ten-year Kaplan-Meier analysis of outcome of patients undergoing postoperative radiotherapy according to fractionation of treatment by (a) overall survival, (b) disease-specific survival, and (c) disease-free survival. Hypofractionated RT: red line; conventional RT: blue line. RT, radiotherapy.

chemotherapy in locally advanced disease is currently being evaluated in clinical trials.

Conventional fractionation consisting of 1.8 to $2.0 \mathrm{~Gy}$ per fraction up to 60 to 70 Gy over 6 to 7 weeks using intensitymodulated radiation therapy is currently the standard scheme to deliver radiotherapy. Melanoma cells have a high capacity for repair of sublethal DNA damage with a low $\alpha / \beta$ ratio. ${ }^{17}$ Based on this, some authors have stressed the use of hypofractionation. ${ }^{18,19}$ A prospective randomized trial conducted by the Radiation Therapy and Oncology Group ${ }^{20}$ compared the standard versus hypofractionated dose regimen for cutaneous melanomas. They were unable to find any difference in survival for the two groups. Likewise, our data showed no survival advantage or significant difference in recurrence rates between patients treated with hypofractionated and conventional fractionation. Owing to the risk of damage to adjacent vital structures (e.g., the optic nerve, orbits, brain) and the risk of toxic effects with high doses per fraction, if hypofractionation is to be considered, highly conformal techniques (intensity-modulated radiation therapy, stereotactic radiotherapy) or proton therapy must be considered.

Two-thirds of the patients in our study experienced recurrence, most having distant metastasis. Most patients with mucosal melanoma have micrometastases at the time of diagnosis of the primary tumor. ${ }^{21}$ Because of this, surgery and radiotherapy may have limited effect on overall prognosis, as they only address local control of the disease. Subgroup analysis of patients with head and neck mucosal melanoma included in a prospective single institution study from China indicated a survival benefit for chemotherapy in these patients. ${ }^{22}$ A second, retrospective study from Korea suggested a survival benefit of adjuvant chemotherapy without any treatment-related deaths. ${ }^{6}$ While these studies suggested that there may be a role for adjuvant chemotherapy, our data did not suggest survival benefits in patients treated with either adjuvant chemotherapy or neoadjuvant chemotherapy. The lack of survival benefits might be related to a genetically distinct disease compared with the Chinese cohort (e.g., $B R A F, N R A S, K I T)$ or due to a locally advanced disease in patients selected for adjuvant or neoadjuvant chemotherapy. Nevertheless, the retrospective nature of our study and the small sample size of patients treated systemically limit our ability to infer meaningful conclusions. It is possible that novel therapies (e.g., ipilimumab, anti-programmed death-1/programmed death ligand-1 [PD1-PDL1 axis], and c-Kit inhibitors) may have a significant role in the treatment of SNMM. ${ }^{23,24}$ Further study is needed due to the rarity and molecular diversity of the disease, and future clinical trials should be designed in a multicenter fashion to be adequately powered to generate meaningful results.

This study has several strengths including a large sample size and homogeneity of the data. However, several limitations must be acknowledged. First, we relied on retrospectively collected data. Treatments were not assigned in a randomized fashion but altered according to extent and progression of disease. Therefore, groups receiving surgery only may represent patients with less-aggressive disease compared with those undergoing adjuvant therapy. As well, we lacked data on radiation planning and could not exclude the possibility that radiation treatment fields may differ between subsites, particularly the neck. Finally, we lacked data on adjuvant treatment toxicity limiting our ability to better define the risk-benefit relationship of adjuvant therapy.

In conclusion, our results show no survival benefits in patients with SNMM treated with PORT, POCRT, or neoadjuvant chemotherapy with PORT. As well, hypofractionation is not associated with survival advantage compared with conventional fractionation. Although paranasal sinus involvement alone should not be an indication for adjuvant therapy, these patients fared significantly worse. In the lack of survival benefits, the role of these treatment modalities in combination with novel agents should be further defined in a clinical trial setting. 
Table 2 Univariate and multivariate analyses for overall survival and disease-specific survival

\begin{tabular}{|c|c|c|c|c|c|c|c|}
\hline \multirow{3}{*}{\multicolumn{2}{|c|}{ Variable }} & \multicolumn{3}{|c|}{ Analysis of OS } & \multicolumn{3}{|c|}{ Analysis of disease-specific survival } \\
\hline & & \multirow{2}{*}{$\begin{array}{l}\text { Univariate } \\
p \text {-Value }\end{array}$} & \multicolumn{2}{|c|}{ Multivariate } & \multirow{2}{*}{$\begin{array}{l}\text { Univariate } \\
p \text {-Value }\end{array}$} & \multicolumn{2}{|c|}{ Multivariate } \\
\hline & & & $p$-Value & $\mathrm{HR}(95 \% \mathrm{Cl})$ & & $p$-Value & $\mathrm{HR}(95 \% \mathrm{Cl})$ \\
\hline \multirow[t]{2}{*}{ Gender } & Male & \multirow[t]{2}{*}{0.84} & \multirow[t]{2}{*}{$\mathrm{N} / \mathrm{A}$} & \multirow[t]{2}{*}{$\mathrm{N} / \mathrm{A}$} & \multirow[t]{2}{*}{0.72} & \multirow[t]{2}{*}{$\mathrm{N} / \mathrm{A}$} & \multirow[t]{2}{*}{$\mathrm{N} / \mathrm{A}$} \\
\hline & Female & & & & & & \\
\hline \multirow[t]{2}{*}{ Age } & $\leq 65 y$ & \multirow[t]{2}{*}{0.04} & \multirow[t]{2}{*}{0.27} & $\begin{array}{l}1 \\
(0.7-2.2)\end{array}$ & \multirow[t]{2}{*}{0.03} & \multirow[t]{2}{*}{0.56} & 1 \\
\hline & $>65 y$ & & & $1.34(0.7-2.2)$ & & & $1.2(0.6-2.3)$ \\
\hline \multirow[t]{2}{*}{ Smoking status } & Never & \multirow[t]{2}{*}{0.04} & \multirow[t]{2}{*}{0.04} & \multirow{2}{*}{$\begin{array}{l}1(1.0-3.1) \\
1.77(1.0-3.1)\end{array}$} & \multirow[t]{2}{*}{0.22} & \multirow[t]{2}{*}{$\mathrm{N} / \mathrm{A}$} & \multirow[t]{2}{*}{$\mathrm{N} / \mathrm{A}$} \\
\hline & Current/former & & & & & & \\
\hline \multirow[t]{2}{*}{ Alcohol consumption } & Never & \multirow[t]{2}{*}{0.51} & \multirow[t]{2}{*}{$\mathrm{N} / \mathrm{A}$} & \multirow[t]{2}{*}{$\mathrm{N} / \mathrm{A}$} & 0.37 & $\mathrm{~N} / \mathrm{A}$ & $\mathrm{N} / \mathrm{A}$ \\
\hline & Current/former & & & & & & \\
\hline T classification & T3 & 0.22 & $\mathrm{~N} / \mathrm{A}$ & $\mathrm{N} / \mathrm{A}$ & 0.38 & $\mathrm{~N} / \mathrm{A}$ & $\mathrm{N} / \mathrm{A}$ \\
\hline & $\mathrm{T} 4 \mathrm{a}$ & & & & & & \\
\hline & $\mathrm{T} 4 \mathrm{~b}$ & & & & & & \\
\hline $\mathrm{N}$ classification & No & 0.12 & $\mathrm{~N} / \mathrm{A}$ & $\mathrm{N} / \mathrm{A}$ & 0.25 & $\mathrm{~N} / \mathrm{A}$ & $\mathrm{N} / \mathrm{A}$ \\
\hline & $\mathrm{N}+$ & & & & & & \\
\hline Site & Nasal cavity & 0.03 & 0.009 & $1(1.2-4.15)$ & 0.02 & 0.02 & 1 \\
\hline & Paranasal sinuses & & & $2.3(1.2-4.15)$ & & & $2.4(1.13-4.9)$ \\
\hline Treatment & Surgery & 0.73 & $\mathrm{~N} / \mathrm{A}$ & $\mathrm{N} / \mathrm{A}$ & 0.57 & $\mathrm{~N} / \mathrm{A}$ & $\mathrm{N} / \mathrm{A}$ \\
\hline & Neoadj.\& PORT & & & & & & \\
\hline & Surgery + RT & & & & & & \\
\hline & Surgery + CRT & & & & & & \\
\hline Margin status & Negative & 0.02 & 0.43 & $1(0.7-2.1)$ & 0.03 & 0.31 & 1 \\
\hline & Positive & & & $1.24(0.7-2.1)$ & & & $1.4(0.7-2.7)$ \\
\hline Skull base invasion & Present & 0.12 & $\mathrm{~N} / \mathrm{A}$ & $\mathrm{N} / \mathrm{A}$ & 0.13 & $\mathrm{~N} / \mathrm{A}$ & $\mathrm{N} / \mathrm{A}$ \\
\hline & Absent & & & & & & \\
\hline Bone invasion & Present & 0.01 & $\mathrm{~N} / \mathrm{A}$ & $\mathrm{N} / \mathrm{A}$ & 0.43 & $\mathrm{~N} / \mathrm{A}$ & $\mathrm{N} / \mathrm{A}$ \\
\hline & Absent & & & & & & \\
\hline Ulceration & $\begin{array}{l}\text { Present } \\
\text { Absent }\end{array}$ & 0.87 & $\mathrm{~N} / \mathrm{A}$ & $\mathrm{N} / \mathrm{A}$ & 0.5 & $\mathrm{~N} / \mathrm{A}$ & $\mathrm{N} / \mathrm{A}$ \\
\hline Mitosis & $<1 \mathrm{~mm}^{2}$ & 0.65 & $\mathrm{~N} / \mathrm{A}$ & $\mathrm{N} / \mathrm{A}$ & 0.73 & $\mathrm{~N} / \mathrm{A}$ & $\mathrm{N} / \mathrm{A}$ \\
\hline & $\geq 1 \mathrm{~mm}^{2}$ & & & & & & \\
\hline
\end{tabular}

Abbreviations: Cl, confidence interval; CRT, chemoradiation therapy; DSS, disease-specific survival; HR, hazard ratio; N/A, not available; Neoadj, neoadjuvant; OS, overall survival; RT, radiotherapy.

Funding

The University of Texas MD Anderson Cancer Center is supported in part by the National Institutes of Health through Cancer Center Support Grant, P30CA016672.

\section{Conflict of Interest}

None of the authors has conflict of interest.

\section{References}

1 Sun CZ, Li QL, Hu ZD, Jiang YE, Song M, Yang AK. Treatment and prognosis in sinonasal mucosal melanoma: a retrospective ana- lysis of 65 patients from a single cancer center. Head Neck 2014; 36(05):675-681

2 Manolidis S, Donald PJ. Malignant mucosal melanoma of the head and neck: review of the literature and report of 14 patients. Cancer 1997;80(08):1373-1386

3 Gal TJ, Silver N, Huang B. Demographics and treatment trends in sinonasal mucosal melanoma. Laryngoscope 2011;121(09): 2026-2033

4 Moreno MA, Roberts DB, Kupferman ME, et al. Mucosal melanoma of the nose and paranasal sinuses, a contemporary experience from the M. D. Anderson Cancer Center. Cancer 2010;116(09):2215-2223

5 Li W, Yu Y, Wang H, Yan A, Jiang X. Evaluation of the prognostic impact of postoperative adjuvant radiotherapy on head and neck mucosal melanoma: a meta-analysis. BMC Cancer 2015;15:758 
6 Ahn HJ, Na II, Park YH, et al. Role of adjuvant chemotherapy in malignant mucosal melanoma of the head and neck. Oral Oncol 2010;46(08):607-611

7 Bartell HL, Bedikian AY, Papadopoulos NE, et al. Biochemotherapy in patients with advanced head and neck mucosal melanoma. Head Neck 2008;30(12):1592-1598

8 Shiga K, Ogawa T, Kobayashi T, et al. Malignant melanoma of the head and neck: a multi-institutional retrospective analysis of cases in northern Japan. Head Neck 2012;34(11):1537-1541

9 Group Pn. Guidelines for the examination and reporting of head and neck cancer specimens. Yorkshire: Cancer Network: LEEDS 2007;17(12):1-12

10 Edge SB, Fritz AG, Byrd DR, Greene FL, Trotti A, Compton CC. AJCC Cancer Staging Manual. 7th ed. New York, NY: Springer; 2010

11 Temam S, Mamelle G, Marandas P, et al. Postoperative radiotherapy for primary mucosal melanoma of the head and neck. Cancer 2005;103(02):313-319

12 Owens JM, Roberts DB, Myers JN. The role of postoperative adjuvant radiation therapy in the treatment of mucosal melanomas of the head and neck region. Arch Otolaryngol Head Neck Surg 2003;129(08):864-868

13 Krengli M, Masini L, Kaanders JH, et al. Radiotherapy in the treatment of mucosal melanoma of the upper aerodigestive tract: analysis of 74 cases. A rare cancer network study. Int J Radiat Oncol Biol Phys 2006;65(03):751-759

14 Pfister DG, Ang KK, Brizel DM, et al; National Comprehensive Cancer Network. Head and neck cancers, version 2.2013. Featured updates to the NCCN guidelines. J Natl Compr Canc Netw 2013;11(08):917-923

15 Benlyazid A, Thariat J, Temam S, et al. Postoperative radiotherapy in head and neck mucosal melanoma: a GETTEC study. Arch Otolaryngol Head Neck Surg 2010;136(12):1219-1225
16 Thariat J, Poissonnet G, Marcy PY, et al. Effect of surgical modality and hypofractionated split-course radiotherapy on local control and survival from sinonasal mucosal melanoma. Clin Oncol (R Coll Radiol) 2011;23(09):579-586

17 Strojan P. Role of radiotherapy in melanoma management. Radiol Oncol 2010;44(01):1-12

18 Trotti A, Peters LJ. Role of radiotherapy in the primary management of mucosal melanoma of the head and neck. Semin Surg Oncol 1993;9(03):246-250

19 Schmidt-Ullrich RK, Johnson CR. Role of radiotherapy and hyperthermia in the management of malignant melanoma. Semin Surg Oncol 1996;12(06):407-415

20 Sause WT, Cooper JS, Rush S, et al. Fraction size in external beam radiation therapy in the treatment of melanoma. Int J Radiat Oncol Biol Phys 1991;20(03):429-432

21 Chang AE, Karnell LH, Menck HR; The American College of Surgeons Commission on Cancer and the American Cancer Society. The National Cancer Data Base report on cutaneous and noncutaneous melanoma: a summary of 84,836 cases from the past decade. Cancer 1998;83(08):1664-1678

22 Lian B, Si L, Cui C, et al. Phase II randomized trial comparing highdose IFN- $\alpha 2 b$ with temozolomide plus cisplatin as systemic adjuvant therapy for resected mucosal melanoma. Clin Cancer Res 2013;19(16):4488-4498

23 Chapman PB, Hauschild A, Robert C, et al; BRIM-3 Study Group. Improved survival with vemurafenib in melanoma with BRAF V600E mutation. N Engl J Med 2011;364(26): 2507-2516

24 Larkin J, Chiarion-Sileni V, Gonzalez R, et al. Combined nivolumab and ipilimumab or monotherapy in untreated melanoma. N Engl J Med 2015;373(01):23-34 